\title{
Special Issue on Transfer Learning
}

\author{
Daniel Kudenko
}

Published online: 9 January 2014

(C) Springer-Verlag Berlin Heidelberg 2013

Machine learning (ML) has been recognized as central to artificial intelligence (AI) for many decades, if not from the early beginnings of AI. The question of how the things that have been learned in one context can be re-used and adapted in related contexts, however, has only been brought to the attention of the wider ML research community over the past few years. In parallel (and sometimes preceding this), transfer learning has been receiving increasing attention in other research areas, e.g. psychology. In this special issue, we present a snapshot of different perspectives on the issues surrounding transfer learning, that we hope will inspire readers to further exploration of this exciting and challenging resarch field.

The contributions to this special issue are a reflection of the diversity of the research area of transfer learning (TL) and the wide range of application domains. In the first article, Haitham Bou Ammar et al. present a discussion of $\mathrm{TL}$ for reinforcement learning (RL). RL has been a focus of much TL research due to its suitability for autonomous intelligent agents. Bou Ammar et al. suggest that some of the tasks that needed to be carried out by the designer, such as state feature mapping, can be automated.

This discussion article is followed by three papers presenting various applications of TL, namely robotic manipulation (Jan Hendrik Metzen et al.), automated negotiation (Siqi Chen et al.), and computational biology (Christian Widmer et al.).

We are also happy to include in this issue an interview with Peter Stone and Matthew Taylor, both prolific RL researchers and amongst the pioneers of TL for RL.

D. Kudenko $(\square)$

Department of Computer Science, University of York,

York YO10 5GH, UK

e-mail: daniel.kudenko@york.ac.uk
The special issue is rounded off by two research reports. Michael Siebers reports on a project applying TL to plan generation. Marco Ragni and Gerhard Strube look at TL from the cognitive psychology viewpoint, discussing insights into human reasoning and what makes transfer problems difficult to solve for humans.

I would like to thank Ute Schmid for her invaluable help in putting this issue together, and the authors for their contributions.

\section{Content}

\subsection{Technical Contributions}

- Haitham Bou Ammar, Siqi Chen, Karl Tuyls, Gerhard Weiss, Automated Transfer for Reinforcement Learning Tasks

- Jan Hendrick Metzen, Alexander Fabisch, Lisa Senger, José de Gea Fernández, Elsa Andrea Kirchner, Towards Learning of Generic Skills for Robotic Manipulation

- Siqi Chen, Haitham Bou Ammar, Karl Tuyls, Gerhard Weiss, Transfer for Automated Negotiation

- Christian Widmer, Marius Kloft, Xinghua Lou, Gunnar Rätsch, Regularization-based Multi-Task Learning with Applications to Genome Biology and Biological Imaging

1.2 Research Projects

- Michael Siebers, Transfer of Domain Knowledge in Plan Generation. Learning Goal-dependent Annulling Conditions for Actions 
- Marco Ragni, Gerhard Strube, Cognitive Complexity and Analogies in Transfer Learning

\subsection{Interview}

- Interview with Peter Stone and Matthew E. Taylor

\section{Service}

While there are hardly any venues and publications that specifically focus on TL, we provide a list of various venues where TL research has been published and discussed.

\subsection{Projects and Organizations}

Effective Bayesian Transfer Learning, http://www.cs.berkeley.edu/russell/ebtl/

\subsection{Journals}

Journal of Autonomous Agents and Multi-Agent Systems (JAAMAS),

http://www.springer.com/computer/ai/journal/10458

Journal of Machine Learning Research (JMLR), http://jmlr.org

\subsection{Conferences}

Thirteenth International Conference on Autonomous Agents and Multi-Agent Systems (AAMAS), http://aamas2014.lip6.fr/

International Conference on Neural Information Processing (NIPS),

http://nips.cc/Conferences/

\subsection{Workshops}

NIPS 2013 Workshop on New Directions in Transfer and Multi-Task: Learning Across Domains and Tasks, http://nips.cc/Conferences/2013/Program/

AAMAS 2014 Workshop on Adaptive and Learning Agents, http://aamas2014.lip6.fr/workshops.php

\subsection{Summer Schools}

European Agents Summer School (EASS), http://www.inf.kcl.ac.uk/events/easss13/ 